\title{
MOLUSCOS AQUÁTICOS ATUAIS DE ECOSSISTEMAS COSTEIROS EM IMBITUBA, IMARUÍ E LAGUNA, SANTA CATARINA, BRASIL: PARÂMETRO DE CARACTERIZAC̣ÃO PARA PALEOAMBIENTES
}

\author{
Inga Ludmila Veitenheimer-Mendes ${ }^{1}$ \\ Vera Lúcia Lopes-Pitoni ${ }^{2}$
}

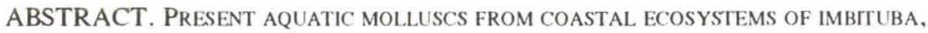
IMARUí AND LAGUNA COUNTIES, SANTA CATARINA, BRAZIL: CARACTERIZATION PARAMETERS FOR PALEOENVIRONMENTS. This paper registers 16 species of aquatic molluscs, especially the limnic and estuarine species Heleobia davisi Silva \& Thomé, 1985. Heleobia australis australis (Orbigny, 1835) and Erodona mactroides Bosc. 1802. from D'Una river and Mirim, Imaruí and Santo Antonio lagoons and Laguna Beach (Santa Catarina, Brazil). This parameter was very important for the paleoecological analysis conduced for that area, where oscillation of the sea level occurred during the Holocen Period. Heleobia davisi is registered for the first time to the Santa Catarina.

KEY WORDS. Molluses, limnic, estuarine, Santa Catarina, Brazil
\end{abstract}

Visando subsidiar os estudos de VEITENHEIMER-MENDES (1993) e LOPESPITONI (1993) sobre paleoambientes holocênicos nos municípios de Imbituba e Imaruí (Santa Catarina), realizaram-se coletas de moluscos em diferentes ambientes aquáticos: foz do rio D’Una, Laguna Mirim, Laguna do Imaruí, Laguna de Santo Antônio e praia de Laguna. Tais coletas fizeram-se necessárias pela inexistência de registros atuais de moluscos e de biota acompanhante naqueles ambientes límnicos e estuarinos, que pudessem ser utilizados para comparação com as espécies de moluscos e fauna acompanhante identificadas pelas autoras acima citadas, em sedimentos holocênicos provenientes de testemunhos de sondagem próximos dos ambientes da coleta atual. Moluscos marinhos atuais da costa brasileira, incluindo a de Santa Catarina, são registrados por RIOS (1994).

\section{MATERIAL E MÉTODOS}

Nos dias 07-08-V-1992, efetuaram-se amostragens qualitativas e quantitativas de moluscos e biota acompanhante em Santa Catarina. As qualitativas foram na Laguna Mirim (Pontos 1 e 3), no rio D’Una próximo à foz com a Laguna Mirim (Ponto 2), na Laguna do Imaruí (Pontos 4 e 5), na Laguna de Santo Antônio junto

1) Departamento de Zoologia, Universidade Federal do Rio Grande do Sul. Av. Paulo da Gama, prédio 12105. 90040-060 Porto Alegre, Rio Grande do Sul, Brasil. Bolsista do CNPq.

2) Museu de Ciências Naturais. Fundação Zoobotânica do Rio Grande do Sul. Caixa Postal 1188. 90001-970 Porto Alegre, Rio Grande do Sul, Brasil. Bolsista do CNPq. 
à barra (Ponto 6) e na praia de Laguna (Ponto 7) (Fig. 1). Para essa amostragem utilizaram-se: coletor de moluscos adotado por VEITENHEIMER- MENDES et al. (1992), para a malacofauna ocorrente na vegetação aquática; peneira para a coleta de bentos; exame de macrófitas, matacões, barranca à beira das lagunas e molhe; tubo amostrador para busca de sedimento nos pontos em maior profundidade e coleta manual. As amostragens quantitativas foram feitas: uma junto à margem nordeste da Laguna Mirim (Ponto 1) com uma peneira de $22 \mathrm{~cm}$ de diâmetro por $3 \mathrm{~cm}$ de altura e $1 \mathrm{~mm}^{2}$ de abertura de malha para cavar e retirar uma camada de sedimento com $10 \mathrm{~cm}$ de espessura aproximadamente, a uma profundidade de $42 \mathrm{~cm}$ em relação à superfície da água, preenchendo totalmente a peneira, e a outra na Laguna do Imaruí (Ponto 4), com um tubo amostrador de ferro de 9,2cm de diâmetro, $28 \mathrm{~cm}$ de altura e $258 \mathrm{~cm}^{3}$ de capacidade, sendo a coluna de sedimento, obtida a $94 \mathrm{~cm}$ de profundidade, equivalente ao tubo totalmente preenchido. $\mathrm{O}$ sedimento das duas amostragens quantitativas foi lavado no próprio local através de uma peneira de $1 \mathrm{~mm}^{2}$ de abertura de malha. Todos os moluscos vivos, obtidos com o emprego das diferentes técnicas de coletas, foram acondicionados em recipientes contendo água do local e transportados no gelo para o laboratório, em caixa com isolamento térmico. No laboratório foram mantidos em refrigerador até a triagem e identificação, por cerca de cinco dias. Esse procedimento de transporte e manutenção foi utilizado visando manter os moluscos vivos para a sua correta identificação, anestesia e fixação.

Durante as amostragens quantitativas, às 10:00 horas na Laguna Mirim e às 14:30 horas na Laguna do Imaruí, foram colhidas amostras de água para determinação da salinidade e amostras de sedimento para análise granulométrica. Estas análises foram executadas pelo Instituto de Pesquisas Hidráulicas (IPH) e Centro de Estudos Costeiros (CECO) da Universidade Federal do Rio Grande do Sul (UFRGS), respectivamente. Os moluscos do gênero Heleobia foram identificados pela malacóloga Maria Cristina Pons da Silva e a vegetação pela Dra. Teresia Strehl. Os moluscos encontram-se depositados na Coleção de Moluscos do Museu de Ciências Naturais (MCN) da Fundação Zoobotânica do Rio Grande do Sul.

\section{RESULTADOS E DISCUSSÃO}

Os resultados qualitativos estão expressos na tabela I, abrangendo todo o conjunto de ambientes explorados. Os resultados das amostras quantitativas e dados abióticos aferidos na Laguna Mirim e do Imaruí encontram-se na tabela II.

Os registros atuais reforçam as deduções de LOPES-PITONI (1993) e VEITENHEIMER-MENDES (1993) de que os ambientes pretéritos daquela região teriam uma ligação mais direta com o mar, com um teor maior de salinidade em relação aos atuais das Lagunas Mirim e do Imaruí. Segundo M. Cristina Pons da Silva (informação verbal) Heleobia autralis nana (Marcus \& Marcus, 1963), registrada por LOPES-PITONI (op. cit.) e VEITENHEIMER-MENDES (op. cit.), caracteriza-se por viver em ambientes estuarinos e de águas salobras, suportando salinidades mais elevadas que $H$. australis australis (Orbigny, 1835) e Heleobia 


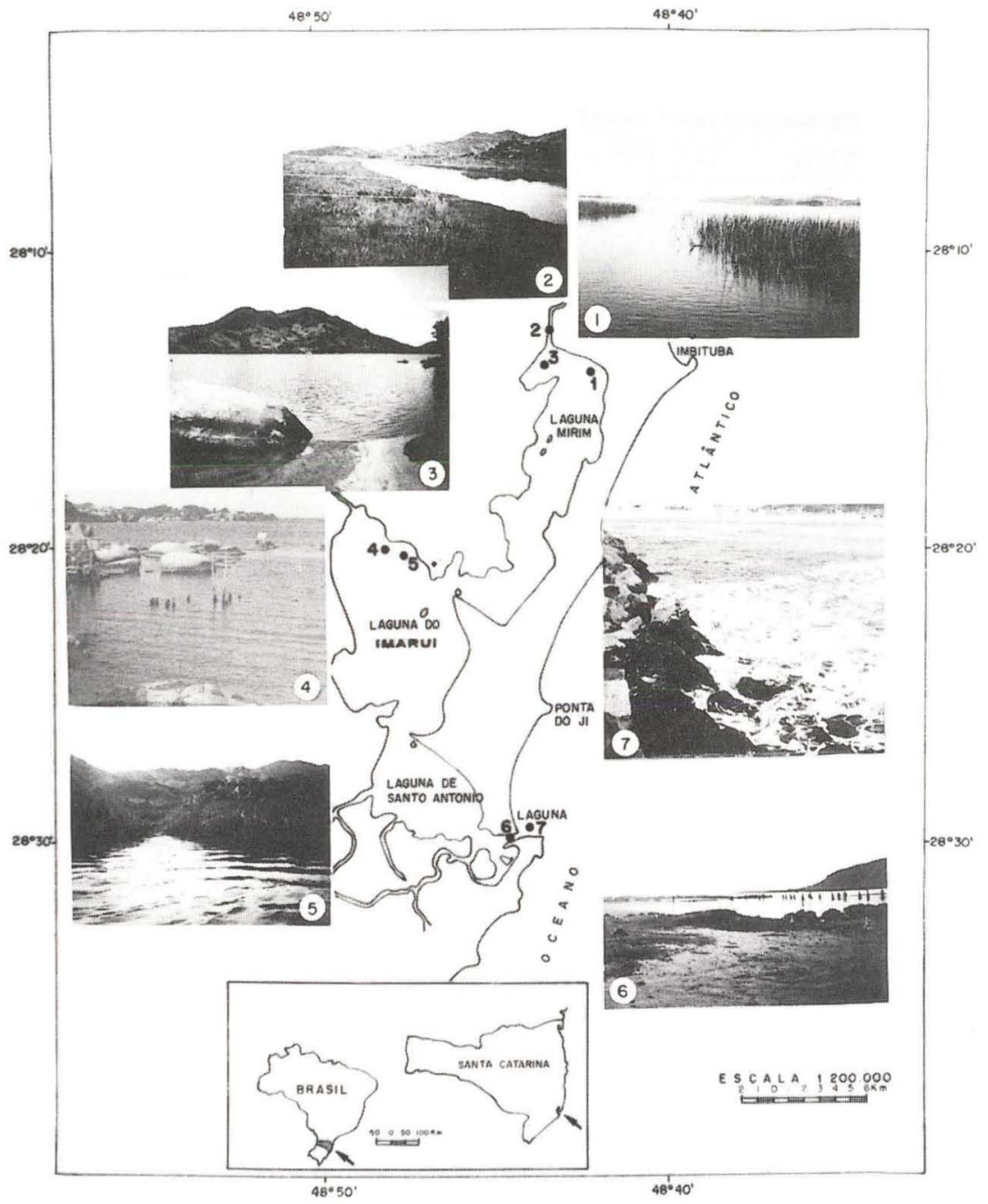

Fig. 1. Locais das coletas de moluscos em ecossistemas costeiros de Santa Catarina, em 07-08-V-1992: Laguna Mirim (Pontos 1 e 3), rio D’Una (Ponto 2), Laguna do Imaruí (Pontos 3 e 4), Laguna de Santo Antônio junto à Barra (Ponto 6) e na praia de Laguna (Ponto 7).

davisi Silva \& Thomé, 1985, espécies registradas na coleta atual, juntamente com Erodona mactroides Bosc, 1802 (Tab. II). O encontro de H. davisi constitui-se no primeiro registro da espécie para Santa Catarina. M. Cristina Pons da Silva (informação verbal) constatou a partir de trabalhos de campo na década de 1980, H. australis australis, $H$. davisi e E. mactroides na parte norte da Laguna dos Patos (Rio Grande do Sul) onde às vezes a salinidade chega a zero, enquanto que mais para o sul, chegando à foz da referida Laguna, observou apenas a ocorrência de $H$. australis nana juntamente com E. mactroides. E. mactroides, segundo 
Tabela I. Amostragem qualitativa de moluscos e biota acompanhante do Rio D'Una (Ponto 2), Laguna Mirim (Pontos 1 e 3), Laguna do Imaruí (Pontos 4 e 5), Laguna de Santo Antônio (Ponto 6) e praia de Laguna (Ponto 7), Santa Catarina, nos dias 07-08-V-1992.

Táxons

Ponto 1 Ponto 2 Ponto 3 Ponto 4 Ponto 5 Ponto 6 Ponto 7

\section{GASTROPODA}

Fissurela rosea

Heleobia australis australis

Heleobia davisi

Odostomia laevigata

\section{Bivalvia}

Amiantis purpuratus

Anadara brasiliana

Anomalocardia brasiliana

Brachidontes exustus

Brachidontes solisianus

Crassostrea rhizophorae

Erodona mactroides

Lucina pectinata

Macoma constricta

Ostrea equestris

Perna perna

Pitar rostratus

Crustacea

Cirripedia (balanídeos)

Decapoda (caranguejos)

VEGETAC̄̃o

Ceratophylum cf. submersum

Cyrpus sp.

Bacopa monnieri

Poacea

$\begin{array}{lll}x & x & x^{*} \\ x^{*}\end{array}$

$X^{*}$

$x^{*}$

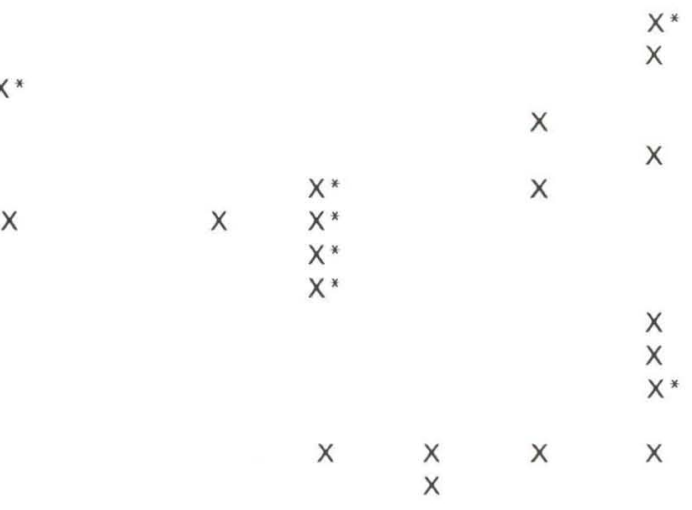

$\mathrm{X}$

$x$

$x$

Potamogeton sp.

(X) Presença, $\left({ }^{*}\right)$ valves ou conchas vazias.

CosTA (1971), é típica de ambientes mixohalinos, sendo encontrada na desembocadura de rios, lagunas e baías do sul do Brasil, Uruguai e Argentina no Oceano Atlântico, com tendência a estar ausente nas zonas pré-límnica e límnica.

O registro dos Ostreidae Crassostrea rhizophorae (Guilding, 1828) e Ostrea equestris Say, $1834 \mathrm{em}$ ambiente de contato direto com o mar em Laguna (Tab. I), a ocorrência de $C$. rhizophorae em bóias na região costeira de Tramandaí (Rio Grande do Sul) em salinidade de $27,7 \mathrm{~g} / 1$ (RIOS et al. 1979), além dos registros de Rios (1994), mostram que estas espécies de Ostreidae são marinhas rasas e que suportam influência de águas continentais.

Dos moluscos registrados nos diferentes corpos d'água, as seguintes espécies foram também identificadas por VEITENHEIMER-MENDES (1993) em sedimentos de testemunhos de sondagem provenientes da região do rio D'Una: Anomalocardia brasiliana (Gmelin, 1792), C. rhizophorae, O. equestris e Pitar rostratus (Koch, 1844); e por LOPES-PITONI (1993) em sedimentos de testemunhos de sondagem de área junto a Laguna do Imaruí: Anomalocardia brasiliana, $C$. rhizophorae, $O$. equestris e $E$. mactroides. 
Tabela II. Amostragem quantitativa de moluscos, escamas de peixe e sementes, presença de detritos e parâmetros físico-químicos na Laguna Mirim (ponto 1) e Laguna do Imaruí (Ponto 4), Santa Catarina, em 07-V-1992.

\begin{tabular}{lcc}
\hline \multicolumn{1}{c}{ Táxons e estruturas } & Exemplares Ponto 1 & Exemplares Ponto 4 \\
\hline Heleobia australis australis & 160 & $12^{* *}$ \\
Heleobia davisi & 175 & $1 * *$ \\
Odostomia laevigata & $19 *$ & $2{ }^{*}$ \\
Erodona mactroides & 7 & \\
Escamas de peixe & 11 & \\
Sementes & $\mathrm{X}$ & 14,50 \\
Detritos vegetais & 2,90 & \\
\hline SALINIDADE (\%o) & & \\
GRANULOMETRIA (\%) & 98,61 & 68,00 \\
$\quad$ Areia & 0,07 & 32,00 \\
$\quad$ Silte & 1,32 & 16,00 \\
$\quad$ Argila & 8,75 & \\
MATÉRIA ORGÂNICA (\%) & & \\
\hline
\end{tabular}

(X) Presença, $\left({ }^{*}\right) 14$ exemplares +5 valves, $\left({ }^{*}{ }^{*}\right)$ somente conchas.

O registro atual de balanídeos e seu respectivo aumento em número de indivíduos a partir da Laguna do Imaruí em direção à praia de Laguna, em decorrência do aumento da oceanicidade (Tab. I), mostrou-se, também, um elemento auxiliar para as deduções dos paleoambientes da região. Durante as coletas pode ser facilmente constatado o aumento no número de balanídeos à medida que se fazia o deslocamento no sentido lagunas-mar, ou seja, baixa salinidade-alta salinidade, uma vez que a presença pontual dos balanídeos, com dimensões reduzidas na Laguna do Imaruí (Ponto 4), passou a constituir, paulatinamente, verdadeiros aglomerados de indivíduos, com maior tamanho, em substratos junto à linha da água até o oceano Atlântico. Esses crustáceos foram observados presos em diferentes substratos, tais como Scirpus sp., matacões, pedras, estacas de madeira. O encontro de balanídeos (Tab. I), de escamas de peixes, sementes e detritos vegetais (Tab. II) no ambiente de ocorrência dos moluscos coincidiram com a biota acompanhante registrada por LOPES-PITONI (1993) e VEITENHEIMERMENDES (1993) nos furos de sondagem da região do rio D'Una e da Laguna do Imaruí.

As demais espécies de moluscos registradas na tabela I são de ambientes marinhos rasos concordando com RIOS (1994), sendo que as conchas de Odostomia laevigata (Orbigny, 1842), Lucina pectinata (Gmelin, 1791) e Macoma constricta (Bruguière, 1792) encontradas na Laguna do Imaruí, provavelmente foram transportadas com as correntes de maré, tendo ocorrido o mesmo com as conchas de Anomalocardia brasiliana coletadas na Laguna Mirim. Anadara brasiliana (Lamarck, 1819), segundo Rios (1994), ocorre em substrato arenoso e de cascalho, semelhante ao substrato no qual foi coletada.

Tanto no Ponto 6 como no Ponto 7 (Tab. I) os ostreídeos C. rhizophorae 
e O. equestris e os mitilídeos Brachidontes exustus (Linnaeus, 1758), Brachidontes solisianus (Orbigny, 1846) e Perna perna (Linnaeus, 1758) encontravam-se aderidos às pedras dos molhes. Os exemplares de $H$. davisi obtidos no rio D'Una (Ponto 2, Tab. I) estavam no fundo arenoso e associadas à Potamogeton sp.; na Laguna Mirim (Ponto 3, Tab. I) encontravam-se fixas à matacões, enquanto exemplares desta mesma espécie e de $H$. australis australis foram coletadas na Laguna Mirim (Ponto 1, Tabs I e II) tanto associadas a Ceratophyllum cf. submersum Linnaeus, Bacopa monnieri (Linnaeus) Penn. e Poacea, como sobre o fundo arenoso.

\section{REFERÊNCIAS BIBLIOGRÁFICAS}

CosTA, C.M.B. 1971. Importância paleoecológica e estratigrática de Erodona mactroides Daudin (Mollusca, Bivalvia). Iheringia, série Geologia, Porto Alegre, 4: 3-18.

LOPES-PITONI, V.L. 1993. Moluscos cenozóicos de subsuperfície em Imaruí, Santa Catarina, Brasil: paleoecologia, transgressões e regressões. Tese de Doutorado, não publicada, Instituto de Geociências, Universidade Federal do Rio Grande do Sul, Porto Alegre, 269p.

RIos, E.C. 1994. Seashells of Brazil. Rio Grande, Universidade do Rio Grande, $2^{a}$ ed., 368p.

Rios, E.C.; V.L. LoPes-PITONI \& I.L. VeITENHEIMER-Mendes. 1979. Moluscos marinhos em bóias no Rio Grande do Sul, Brasil. Publ. Avuls Fund. Zoobotânica, Porto Alegre, 4: 103-107.

VEITENHEIMER-MENDES, I.L. 1993. Malacofauna, paleoecologia e biofácies de sedimentos holocênicos da planície costeira de Imbituba e Imaruí, Santa Catarina, Brasil. Tese de Doutorado, não publicada, Instituto de Geociências, Universidade Federal do Rio Grande do Sul, Porto Alegre, 309p.

VeITENheimer-Mendes, I.L.; V.L. LOPEs-PItoni; M.C.P. DA Silva; J.E. AlmEIDA-CAON \& N.T. SChroder-PFEIFFER. 1992. Moluscos (Gastropoda e Bivalvia) ocorrentes nas nascentes do rio Gravataí, Rio Grande do Sul, Brasil. Iheringia, série zoologia, Porto Alegre, (73): 69-76.

Recebido em 30. VIII. 1994; aceito em 05. IX. 1995. 